\title{
Exclusive measuring system of constitutive parameters for living soft tissue and application to facial aging problems
}

\author{
T. Tsuta ${ }^{1}$, T. Iwamoto ${ }^{2} \&$ T. Fujimura ${ }^{3}$ \\ ${ }^{1}$ Hiroshima International University, Japan \\ ${ }^{2}$ Hiroshima University, Higashi-Hiroshima, Japan \\ ${ }^{3}$ Shiseido Ltd., Shinagawa, Tokyo, Japan
}

\begin{abstract}
An automatic loading and sensing system for obtaining in vivo multi-axial $\mathrm{P}_{i}-\delta_{i}$ $(i-1,2, \ldots$,$) curves in facial skin of living humans has been developed. Once the$ $\mathrm{P}_{i}-\delta_{i}$ curves are obtained through a series of experiments for respective directions $(\mathrm{x}-\mathrm{x}, \mathrm{y}-\mathrm{y}, \mathrm{x}-\mathrm{y}, \ldots$,$) , then the constitutive parameters are identified by the least$ squares approach, using the analogy with the FEM computer model, so that the gap between the experimental $\mathrm{P}_{i}-\delta_{i}$ curves and the FEM $\mathrm{P}_{i}-\delta_{i}$ curves can be minimized in respective $i$-th directions by improving the physical parameters. Application to the forehead skins in actual humans aged 22 years and 54 years has been examined. Finally the constitutive parameters of facial muscles have also been identified. These results are applied to generate facial wrinkling and permanent wrinkling problems induced by aging. It is clarified that earlier wrinkling in older aged humans is mainly induced by the change in constitutive parameters.

Keywords: in vivo skin constitutive model, sensing of multiaxial $P-\delta$ curve, analogy to FEM computer model, forehead skin, wire-driven remote loading hardware system, motion control system, least squares identification approach, some applications.
\end{abstract}

\section{Introduction}

The combined contraction of about thirty kinds of facial muscles induces numerous types of facial expression and wrinkling. It induces permanent 
wrinkling in the human face due to aging, and induces serious problems from the view point of beauty science to the human face.

The authors have studied so far this kind of problem, with respect to the finite element formulation of facial skin with hyperelastic and finite deformation characteristics (7), post buckling analysis of facial wrinkling (9), the formulation of contraction mechanics in facial muscles (4), and the modeling of permanent wrinkling under iterative residual strain generated (8) in the wrinkle bottom due to creep-relaxation mechanics after facial expressions.

This paper presents the design and development of a handy type of automatic loading and sensing system for obtaining in vivo multi-axial load-displacement $\left(\mathrm{P}_{i}-\delta_{i}\right)$ curves in the facial skin of a living human. By combining the experimental $\mathrm{P}_{i}-\delta_{i}$ curves with those of FEM computer modeling, the constitutive parameters in the multi-axial stress-strain relations of facial skin are determined using the least squares approach.

The constitutive parameters of facial muscles have also been identified. Those results are applied to generate facial wrinkling and permanent wrinkling problems, induced by aging.
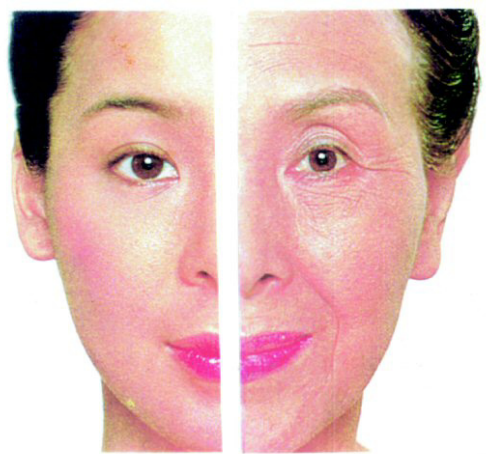

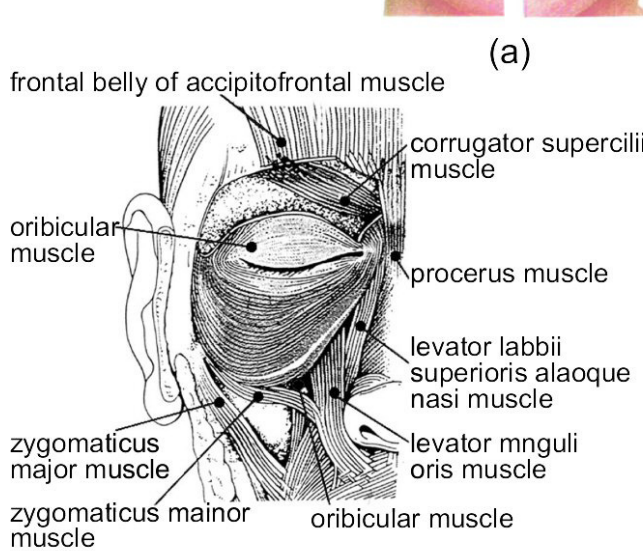

(b)

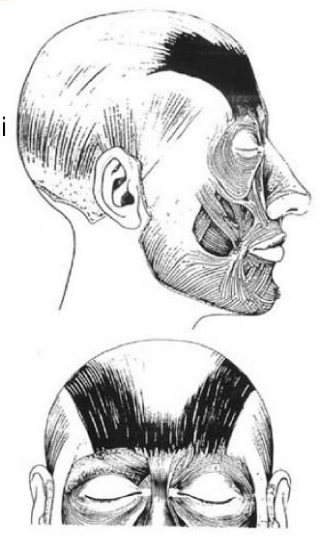

(c)

Figure 1: The constitutive parameters of facial muscles. 


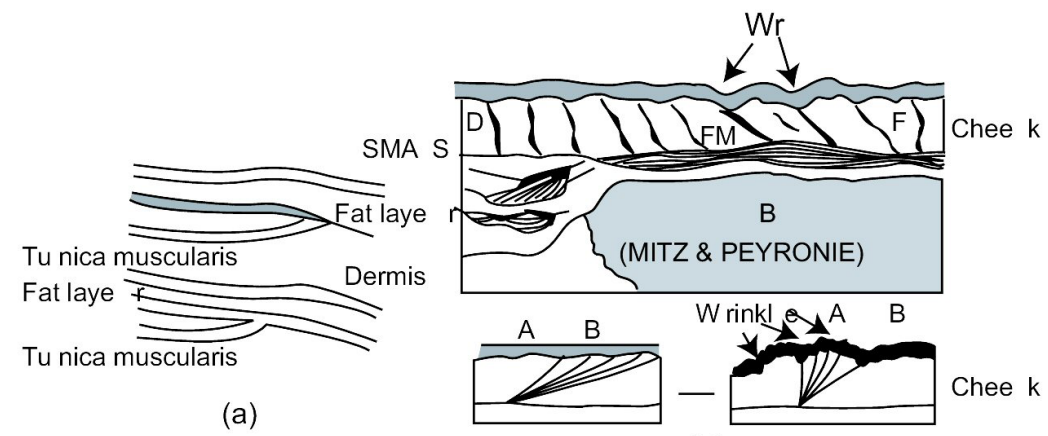

(b)
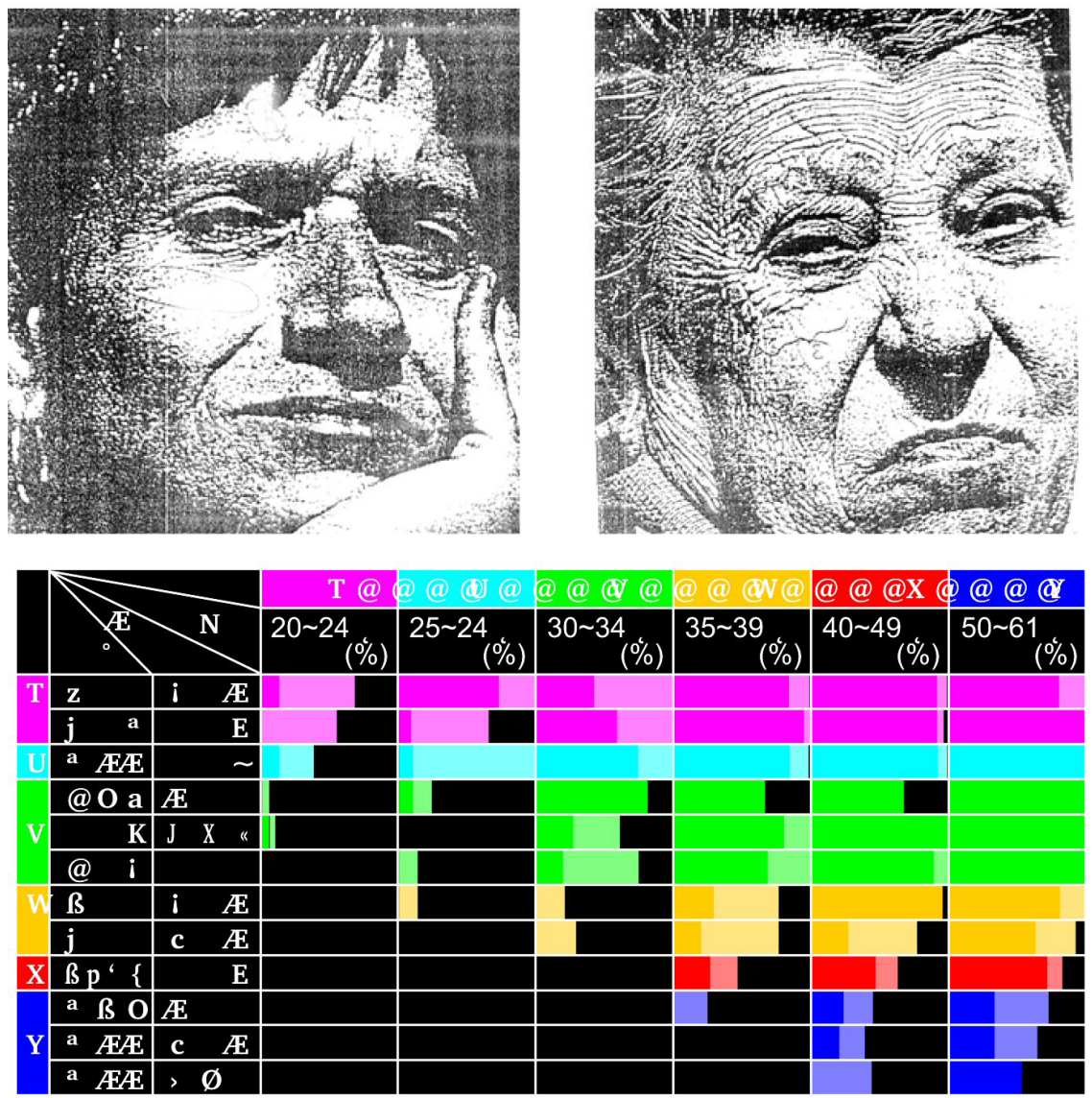

Figure 2: $\quad$ Ultrasonic tomography figure of the cheek subcutaneous tissue. 


\section{Constitutive equation of skin}

\subsection{Green strain and Kirchoff stress}

Since skin behavior involves large strain and finite deformation, it is necessary to formulate it using Green strain as

$$
E_{i j}=\frac{1}{2}\left[\frac{\partial u_{i}}{\partial X_{j}}+\frac{\partial u_{j}}{\partial X_{i}}+\frac{\partial u_{m}}{\partial X_{i}} \frac{\partial u_{m}}{\partial X_{j}}\right]
$$

where $u_{i}$ is displacement, $X_{i}$ the coordinate before deformation and $i, j, k=1,2,3$ respectively. The summation rule is adopted.

The strain energy function $\mathrm{W}$ is expressed by using the invariants of Green strain $E_{i j}$, i.e.,

$$
J_{1}=E_{i i}, \quad J_{2}=E_{i i} E_{j j}-E_{i j} E_{j i}, \quad J_{3}=\operatorname{det} E_{i j}
$$

as

$$
W=W\left(J_{1}, J_{2}, J_{3}\right)
$$

then, the Kirchoff stress $S_{i j}$ can be written by

$$
S_{i j}=\frac{\partial W}{\partial E_{i j}}=\sum_{k=1}^{3} \frac{\partial W}{\partial J_{k}} \cdot \frac{\partial J_{k}}{\partial E_{i j}}
$$

The incompressibility condition of skin can be written by

$$
I_{3}-1=2 J_{1}+4 J_{2}+8 J_{3}=0
$$

where $I_{3}$ is the third invariant of Green's deformation tensor.

If the incompressibility condition is introduced into Eq.(4) by using the Lagrange parameter $h$, then we have

$$
S_{i j}=\frac{\partial W}{\partial E_{i j}}+h \frac{\partial I_{3}}{\partial E_{i j}}
$$

where $h=S_{i j} / 3$ is the static pressure component of the stress.

In order to obtain the skin constitutive equation, the strain energy function $W$ is expressed in the following form, using the evolutional relation of Fung (1993).

$$
W=F(E)+\frac{1}{2}\left[C_{s}\right] \exp [G(E)]
$$

where

$$
F(E)=\frac{1}{2}\{E\}^{T}\left[D_{A}\right]\left\{E_{1}\right\}
$$

Substituting Eq.(7) into (4), the following constitutive equations are obtained by assuming transverse isotropy

$$
\{S\}=[D]\{E\}=\left(D_{A}+C_{S} D_{B} \exp (G)\right)\{E\}
$$

or 


$$
\begin{aligned}
& \left\{\begin{array}{l}
S_{11} \\
S_{22} \\
S_{33} \\
S_{12} \\
S_{23} \\
S_{31}
\end{array}\right\}=\left[\begin{array}{cccccc}
a_{1} & a_{7} & a_{8} & 0 & 0 & 0 \\
a_{7} & a_{2} & a_{9} & 0 & 0 & 0 \\
a_{8} & a_{9} & a_{3} & 0 & 0 & 0 \\
0 & 0 & 0 & a_{4} & 0 & 0 \\
0 & 0 & 0 & 0 & a_{5} & 0 \\
0 & 0 & 0 & 0 & 0 & a_{6}
\end{array}\right]\left[\begin{array}{l}
E_{11} \\
E_{22} \\
E_{33} \\
E_{12} \\
E_{23} \\
E_{31}
\end{array}\right] \\
& +\left[\begin{array}{cccccc}
\mu_{1} & \mu_{7} & \mu_{8} & 0 & 0 & 0 \\
\mu_{7} & \mu_{2} & \mu_{9} & 0 & 0 & 0 \\
\mu_{8} & \mu_{9} & \mu_{3} & 0 & 0 & 0 \\
0 & 0 & 0 & \mu_{4} & 0 & 0 \\
0 & 0 & 0 & 0 & \mu_{5} & 0 \\
0 & 0 & 0 & 0 & 0 & \mu_{6}
\end{array}\right]\left[\begin{array}{l}
E_{11} \\
E_{22} \\
E_{33} \\
E_{12} \\
E_{23} \\
E_{31}
\end{array}\right] \exp [G(E)]
\end{aligned}
$$

where, $a_{i}$ and $\mu_{i}(i=1,2, \ldots, 9)$ are material constants and the material parameter $\left[C_{\mathrm{s}}\right]$, which expresses the transition point from superior zone in first term to superior zone in second term of the right hand side in Eq.(9), can be expressed by

$$
\left[C_{s}\right]=\left[\begin{array}{cccccc}
C_{1} & C_{7} & C_{8} & 0 & 0 & 0 \\
C_{7} & C_{2} & C_{9} & 0 & 0 & 0 \\
C_{8} & C_{9} & C_{3} & 0 & 0 & 0 \\
0 & 0 & 0 & C_{4} & 0 & 0 \\
0 & 0 & 0 & 0 & C_{5} & 0 \\
0 & 0 & 0 & 0 & 0 & C_{6}
\end{array}\right]
$$

We can introduce the incompressibility condition into Eq.(9) using Eq.(6), as

$$
\{S\}=[D]\{E\}+h\left\{\frac{\partial I_{3}}{\partial E_{i j}}\right\}
$$

The two dimensional constitutive equation for plane stress condition can be expressed by using Eq.(9) as

$$
\begin{aligned}
\left\{\begin{array}{l}
S_{11} \\
S_{22} \\
S_{12}
\end{array}\right\} & =\left[\begin{array}{ccc}
a_{1} & a_{4} & 0 \\
a_{4} & a_{2} & 0 \\
0 & 0 & a_{3}
\end{array}\right] \\
& +\left[\begin{array}{ccc}
C_{1} & C_{4} & 0 \\
C_{4} & C_{2} & 0 \\
0 & 0 & C_{3}
\end{array}\right]\left[\begin{array}{ccc}
\mu_{1} & \mu_{4} & 0 \\
\mu_{4} & \mu_{2} & 0 \\
0 & 0 & \mu_{3}
\end{array}\right]\left\{\begin{array}{l}
E_{11} \\
E_{22} \\
E_{12}
\end{array}\right\} \exp [G(E)]
\end{aligned}
$$

Using the incremental form of Eq.(1) and (9), the incremental stiffness equation of the skin can be written in the form of $[k]\{D U\}=\{D P\}+\{R\}$ (Tsuta et al [6]), and can be solved for nodal increments of displacement using FEM. The computer system has been developed and used for solving the identifying problems shown below. 


\section{Automatic loading and sensing system}

\subsection{Basic concept}

Since there is no edge in the facial skin of living humans, an axial end of the follow cylinder is suppressed on the skin surface as shown in Fig.3. Since it generates a disc-like subregion of skin, fixed at the circular boundary, it is used as the test region. The end of the loading bar $\mathrm{A}$ is pasted on a point near the center of the disc skin surface, and the force and displacement are applied on it by moving the bar A to the translational and the rotating directions, as shown in Fig.1(b) and (c) respectively.

In the case of the forehead skin, loading cases are divided into three types, i.e., $i=1$ and $i=2$ for muscle-contractile, direction and the orthogonal to it, and $i=3$ for the rotational directions $(T, \theta)$ as shown in the same figure.

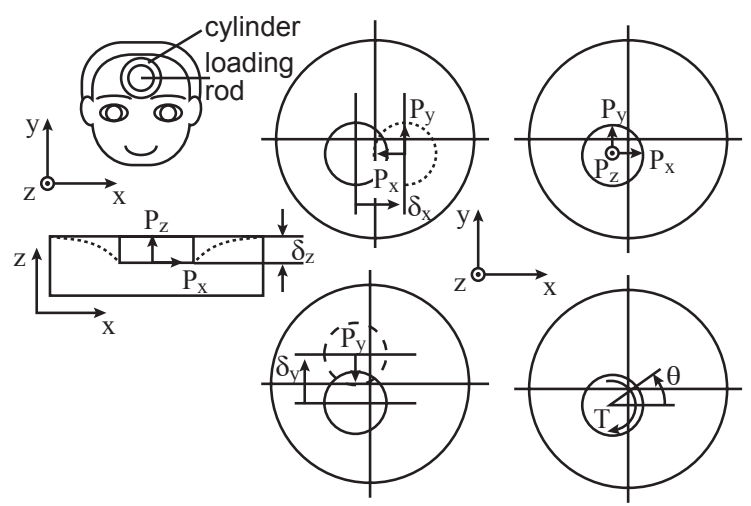

Figure 3: $\quad$ Loading modes and displacement.

\subsection{Construction of the system}

The automatic loading and sensing system consist of Powering subsystem (PS), Wire cable subsystem (WCS), Sensor sub-system (SSS), Motor controller (MC), Programmable controller (PRC), Laser displacement and Force measuring subsystems (Amplifier (AMP) and Amplifier (FA)) and Personal computer (PC). The whole construction is schematically shown in Fig.4 (a) and (b), and a view of measuring a living human is shown in Fig.3. The Sensor Subsystem and Powering Subsystem are connected with the wire cable subsystem, and the force is transmitted from stepping motors into loading bar $\mathrm{A}$, through the wire cable system. The rotation of the motor is controlled by using the program stored in the personal computer.

The Laser and load-cell sensors stored in the SSS can measure the displacements and forces acting to the interface between bar A and the pasted skin.

Below we outline each subsystem. 

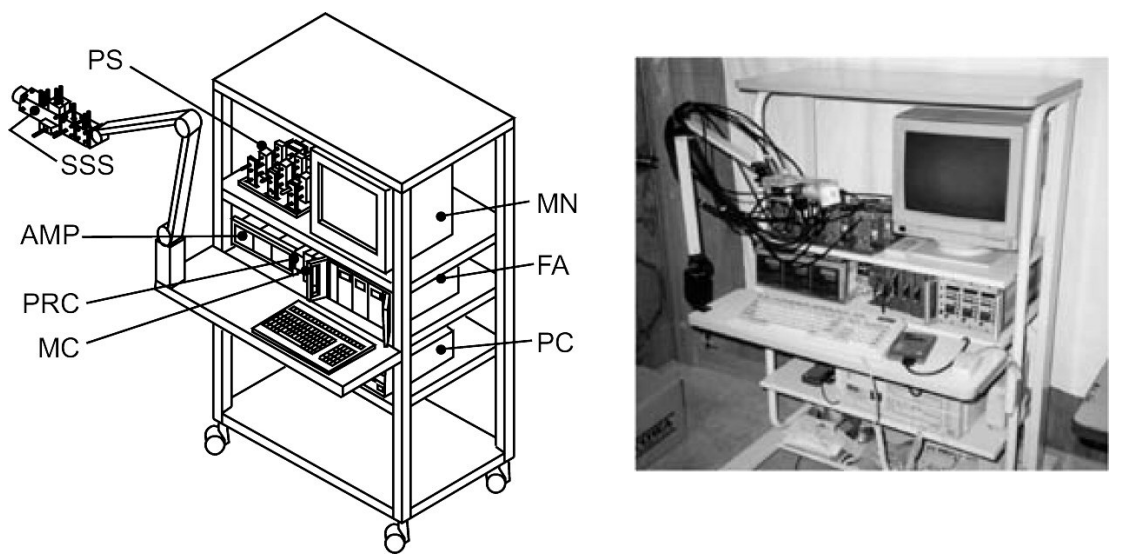

Figure 4: $\quad$ Automatic loading and sensing system of multi-axial $P-\delta$ curves.
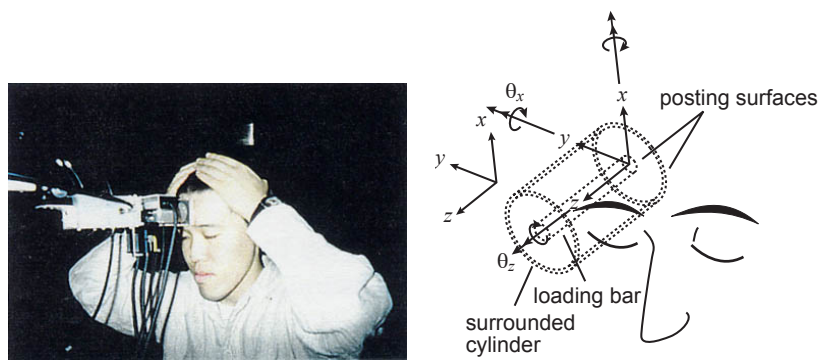

Figure 5: A view of sensor system in measuring of a living human.

\subsection{Loading subsystem}

A schematical view of the sensor subsystem (SSS) is shown in Fig.2(a). The SSS is made of Al-alloy, with a weight of $12 \mathrm{~N}$ and is surrounded by a box-shaped casing with a width of $20 \mathrm{~mm}$, and a length of $200 \mathrm{~mm}$.

The end of the hollow cylinder (90) is attached to the skin by pasting, and the loading bar $\mathrm{A}$ is pasted on the central part of the interior skin, surrounded by the cylinder (90). The bar $\mathrm{A}$ is driven to the $\mathrm{x}, \mathrm{y}$ and $\theta$-directions, respectively, by the powering subsystem (PS) and the wire cable subsystem (16X, 16Y and 16T).

The bearing support 102 for bar $\mathrm{A}$ is movable in the $\mathrm{x}$ direction according to the linear motion bearings 104 and 110, independent to the y-direction motion, and the bearing support 98 for bar $\mathrm{A}$ is movable in the y-direction according to the linear motion bearings 112 and 100, independent to the $\mathrm{x}$-direction motion respectively. The rotating motion of the loading bar A can be given by push-pull motion of 16T wires around the disc guide 116, and it is separated from the other motions to the $\mathrm{x}$ and $\mathrm{y}$ directions by the linear motion bearings 108 and 110 . 


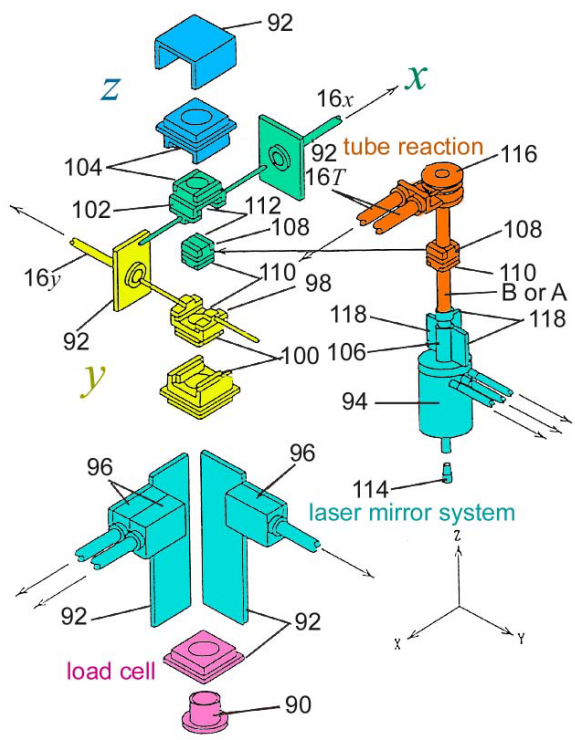

Figure 6: Loading mechanics and measuring facilities inside the sensor subsystem.

\subsection{Powering and motion control subsystems}

The powering subsystem is remote from the SSS as shown in Fig.4 and the power from the stepping motor is transmitted to the loading bar A, through the wire in tube system, as shown in Fig.7. The driving unit is constructed of a stepping motor, ball bush bearing, ball screw and wire-in tube system.

The rotation of the motor is transformed to linear motion by using the ball screw and the force is transmitted to the loading bar A through the wire tube system.
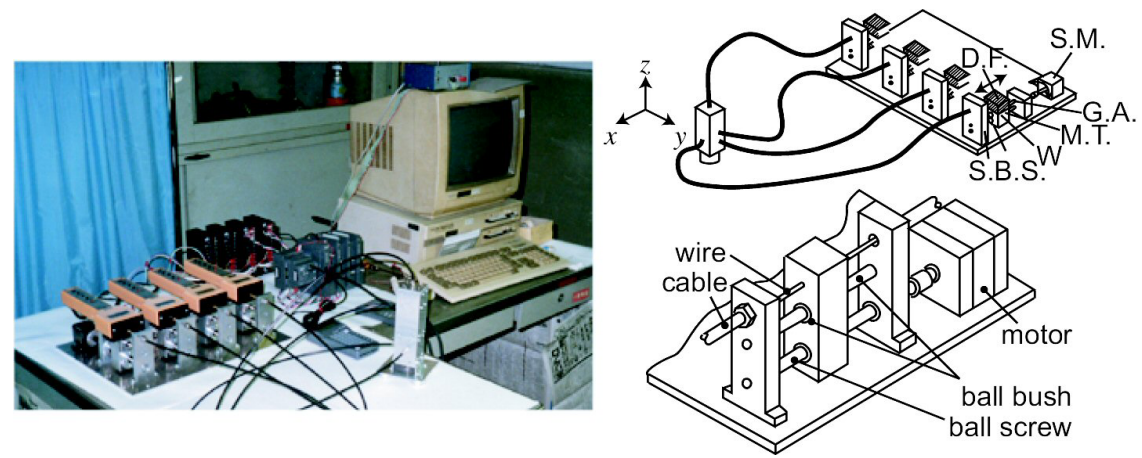

Figure 7: Wire in tube system. 
As shown in Fig.9, the motion of the stepping motor (60) is controlled by the motor driver (80), motor controller (78), programmable controller (76) and computer program stored in the personal computer (74).

The control signal is transmitted to the motor driver using the on/off command of the relay in the sequence circuit (80) and the motor rotates under assigned step number and velocity.

\subsection{Measuring subsystem}

Three set of laser systems $(96,118)$ for measuring the displacements of the loading bar A for $x, y$ and $\theta$ directions are stored in the sensor subsystem at the nearest part to the skin as shown in Fig.6, and three kinds of beams radiated from the laser source are reflected from the reflector plates 118, from which the relative displacement between the pasted cylinder and the pasted loading bar A can be measured under no contacting condition.

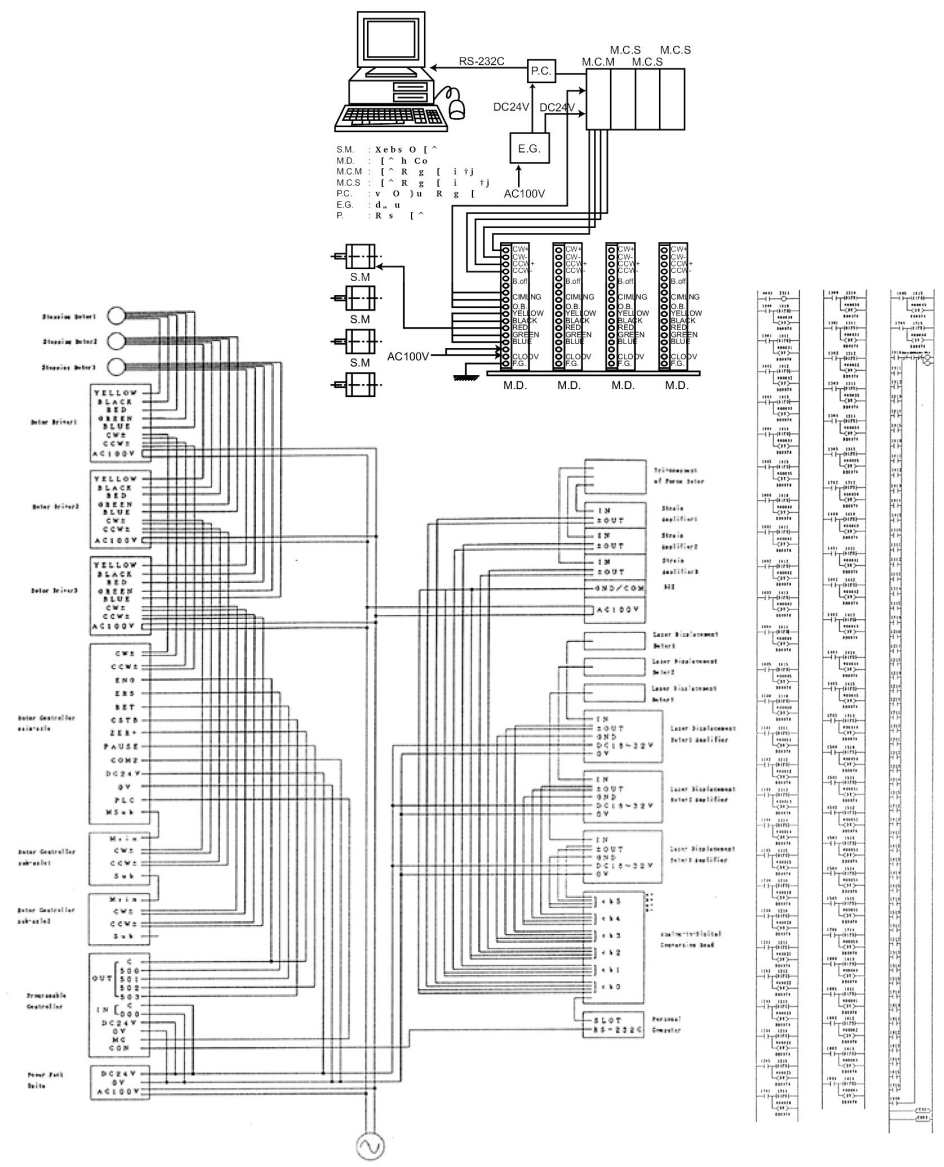

Figure 8: Control system for motor driving and sensing. 
The forces acting on the skin can be measured by using exclusive load-cell system 92 to the $x, y$ and $\theta$ directions, at the same time respectively.

The flow chart of the automatic measurement system for loading and sensing of multi-axial $P-\delta$ curves of skin in living human is shown in Fig.9.

The measured data for forces and displacements are amplified by amplifiers (124) and (128) and converted to digital data at (126). They are stored in the computer system, together with the driving conditions.

In the plane stress condition, the parameters to be identified are $\alpha_{i j}, C_{i j}$ and $\mu_{i j}$ $(i=1 \ldots 4)$ as shown in Eq.(13).

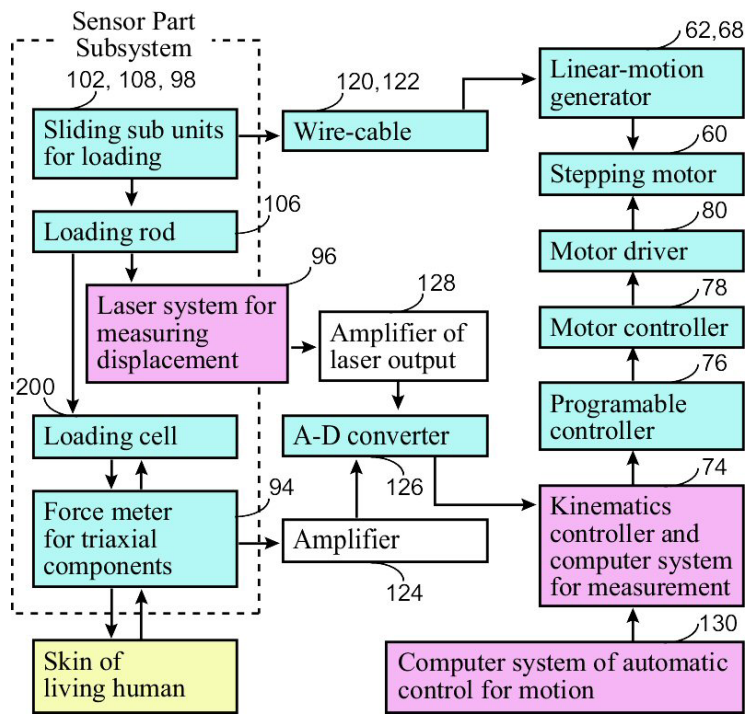

Figure 9: $\quad$ Flow chart of control in automatic loading and sensing subsystem.

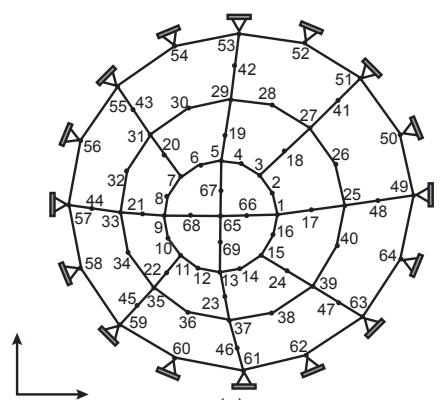

(a)

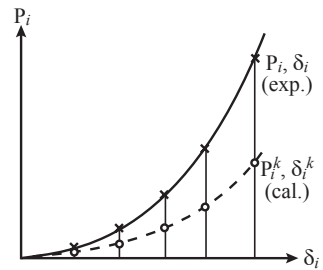

(b)

Figure 10: $\quad$ FEM model and curve fitting. 
The load-displacement curves for determining the diagonal parameters of the constitutive matrix shown in Eq.(13), are to be three types, $P_{x}-\delta_{x}, P_{y}-\delta_{y}$ and $T-\theta$ respectively. The off-diagonal curves, such as, $P_{y}-\delta_{x}$ and $P_{x}-\delta_{y}$ for determining off-diagonal components in Eq.(13) are subsidially obtained from above test by using the offset loading to the cylinder center, with the amount of $e_{x}, e_{y}$ as shown in Fig. 10(a)

\section{Identification of constitutive parameters}

\subsection{Identification scheme}

The finite element system HYEL 2D has been developed for solving the hyperelastic and finite deformation solid, based on the above theory.

The 2D finite element model, similar to that of the experiment, is modeled as shown in Fig.10(a) for obtaining $P-\delta$ curve by giving prescribed constitutive parameters.

Once the multiaxial $P_{i}-\delta_{i}$ curves are obtained from a series of experiments, the constitutive parameters of the Kirchoff stress-Green strain relation can be determined by the least squares approach, such that the gap between the experimental and calculated $P_{i}-\delta_{i}$ curves $(i=1,2, \ldots)$ is minimized by varying the physical parameters appearing in the constitutive equations.

Below we formulate the basic relation for the identification approach.

The displacements, obtained from experiments, are defined to be $\delta_{i}$ and the calculated to be $\delta_{\mathrm{i}}^{k}$ for $k$-th iteration and $i$-th loading mode, and the vectors and $\delta$ are defined to be

$$
\begin{aligned}
& \bar{\delta}=\left\{\bar{\delta}_{1} \bar{\delta}_{2} \bar{\delta}_{3} \cdots \bar{\delta}_{m}\right\}^{T} \\
& \delta^{k}=\left\{\delta_{1}^{k} \delta_{2}^{k} \delta_{3}^{k} \cdots \delta_{m}^{k}\right\}^{T}=\delta\left(\alpha_{k}\right)
\end{aligned}
$$

where $\alpha_{k}$ is constitutive parameter.

Then the steepest descent approach for obtaining material parameters can be given as follows. Since the convergent state shows that each component of the vector $\delta^{k}$ coincides with those of $\delta$, obtained by experiments, the objective function $g_{i}\left(\alpha^{k}\right)$ on the k-th step can be defined to be

$$
g_{i}\left(\alpha^{k}\right)=\bar{\delta}_{i}-\delta_{1}^{k} \rightarrow 0
$$

The objective function $g_{i}\left(\alpha^{k+1}\right) \equiv g_{i}\left(\alpha^{k}+\Delta \alpha\right)$ on the step $k+1$ can be expanded to the Taylor expansion series as

$$
g_{i}\left(\alpha^{k}+\Delta \alpha\right)=g_{i}\left(\alpha^{k}\right)+\sum_{j=1}^{n} \frac{\partial g_{i}\left(\alpha^{k}\right)}{\partial \alpha_{j}} \Delta \alpha_{j}+\cdots
$$

where $\Delta \alpha_{j}$ is the increment of the design parameter and can be given by

$$
\Delta \alpha_{j}=\alpha_{j}^{k+1}-\alpha_{j}^{k}
$$

Then the condition of Eq.(15) on the step $k+1$ can be rewritten as 


$$
g_{i}\left(\alpha^{k}\right)=-\sum_{j=1}^{n} \frac{\partial g_{i}(\alpha)}{\partial \alpha_{j}} \Delta \alpha_{j}=\sum_{j=1}^{n} H_{i j}\left(\alpha^{k}\right) \cdot\left(\alpha_{j}^{k+l}-\alpha_{j}^{k}\right)
$$

where the matrix $H_{i j}$ is the gradient of $g_{i}\left(\alpha^{k}\right)$. Using Eq.(14) and Eq.(16), we obtain

$$
\begin{aligned}
\bar{\delta}_{i}-\delta_{i}\left(\alpha^{k}\right) & =\sum_{j=1}^{n} H_{i j}\left(\alpha^{k}\right)\left(\alpha_{j}^{k+l}-\alpha_{j}^{k}\right) \\
& =\left\{H_{i 1} H_{i 2} \cdots H_{i n}\right\}\left[\begin{array}{c}
\alpha_{1}^{k+1}-\alpha_{1}^{k} \\
\alpha_{2}^{k+1}-\alpha_{2}^{k} \\
\vdots \\
\alpha_{n}^{k+1}-\alpha_{n}^{k}
\end{array}\right]
\end{aligned}
$$

Assembling the above relation to the whole system,

$$
\bar{\delta}-\delta\left(\alpha^{k}\right)=H\left(\alpha^{k}\right) \cdot\left(\alpha^{k+1}-\alpha^{k}\right)
$$

where

$$
H\left(\alpha^{k}\right)=\left[H_{i j}\left(\alpha^{k}\right)\right]=\left[\frac{\partial u_{i}\left(\alpha^{k}\right)}{\partial \alpha_{j}}\right], \quad i=1,2 \cdots
$$

The design variable vector $\alpha^{k}+1$ can be determined from Eq.(18) as

$$
\alpha^{k+1}=\alpha^{k}+\left[H\left(\alpha^{k}\right)\right]^{-1}\left\{\bar{\delta}-\delta\left(\alpha^{k}\right)\right\}
$$

In the actual case, since the component $H_{i j}$ of the gradient matrix $\mathrm{H}$ is nonlinear and cannot be expressed explicitly using the function of $\alpha_{i}$, $\delta^{k}$ is obtained numerically. It is better to calculate $H_{i j}\left(\alpha^{k}\right)$ numerically as in the following form, using numerical simulation by FEM, i.e.

$$
H_{i j}\left(\alpha^{k}\right)=\frac{\partial \delta_{i}\left(\alpha^{k}\right)}{\partial \alpha_{j}}=\frac{\left\{\delta_{i}\left(\alpha^{k}+\Delta \alpha_{j}\right)-\delta_{i}\left(\alpha^{k}\right)\right\}}{\Delta \alpha_{j}}
$$

where

$$
\delta_{i}\left(\alpha^{k}+\Delta \alpha_{j}\right)=\delta_{i}\left(\begin{array}{c}
\alpha_{1} \\
\vdots \\
\alpha_{j}+\Delta \alpha_{j} \\
\vdots \\
\alpha_{n}
\end{array}\right)
$$

\subsection{Generalization using the least squares approach}

In order to identify the calculated $P-\delta$ curves to those of the experiment, it is necessary to use several representative points instead of nonlinear characteristics for a continuous curve, as shown in Fig.10(b). 
Otherwise, it takes enormous times for convergence. So we generalize the above model to include more sufficient parameters $\delta_{i}(m>n)$ than unknown constitutive parameters $\alpha^{k}(n)$, based on the least squares approach.

Assembling the residual of both sides of Eq.(18), the square sum of them can be expressed by

$$
L=\sum_{i=1}^{m}\left\{\sum_{j=i}^{n} H_{i j} \cdot \Delta \alpha_{j}-g_{i}\left(\alpha^{k}\right)\right\}^{2}
$$

The minimizing condition of the above equation can be written by

$$
\frac{\partial L}{\partial \Delta \alpha}=0 \quad(\mu=1,2, \cdots, n)
$$

from which we obtain

$$
\left\{\begin{array}{c}
\Delta \alpha_{1} \\
\Delta \alpha_{2} \\
\cdots \\
\Delta \alpha_{n}
\end{array}\right\}=\left[\begin{array}{cccc}
\Sigma H_{i 1}^{2} & \Sigma H_{i 1} \Sigma H_{i 2} & \cdots & \Sigma H_{i 1} \Sigma H_{i m} \\
& \Sigma H_{i 2}^{2} & \cdots & \Sigma H_{i 2} \Sigma H_{i m} \\
& & \cdots . . & \\
s y m & & & \Sigma H_{i 1}^{2}
\end{array}\right]\left(\begin{array}{c}
\Sigma H_{i 1} g_{i} \\
\Sigma H_{i 2} g_{i} \\
\cdots \\
\Sigma H_{i n} g_{i}
\end{array}\right\}
$$

Several representative points are selected from experimental $P_{i}-\delta_{i}$ curve $(i=x, y, \ldots)$ and define the displacement vector $\delta_{i}$. Then the revised parameter $\alpha_{j}^{k+1}=\alpha_{j}^{k}+\Delta \alpha_{j}$ can be determined from Eq.(25), by giving djk on the step k, and the FEM calculation on the next step $k+1$ can be done. The process is continued up to obtain the convergent solution.

The computer simulation program has been developed to identify the constitutive parameters. The finite element program HYEL is iteratively used for solving the problems by updating the material parameters.

\section{Application}

\subsection{Measurement of $\mathbf{P}-\delta$ curves}

In order to verify the applicability of the method developed, some loading tests using the sensor subsystem have been carried out for a young man aged 22 years.

Lattice networks with one side length of $10 \mathrm{~mm}$ are marked by painting in horizontal and vertical directions on the facial skin as shown in Fig.11.

The thickness of the forehead skin and under-skin tissue are measured at these nodes using the ultrasonic measurement system with $20 \mathrm{MHz}$ and $7 \mathrm{MHz}$ types, made by Aroka Ltd. The obtained thickness of the skin, under-skin and muscle, are shown in Fig.11(b), from which it is seen that the thickness of the skin is nearly uniform around $2 \mathrm{~mm}$. In the next place, the plastic cylinder with inner diameter of $18 \mathrm{~mm}$ is pressed on the forehead skin of the above model A as shown in Fig.3, and the end of the loading bar A is pasted on the skin at offset point $1 \mathrm{~mm}$ apart from the $\mathrm{x}$ and $\mathrm{y}$ directions from the cylinder center respectively. 

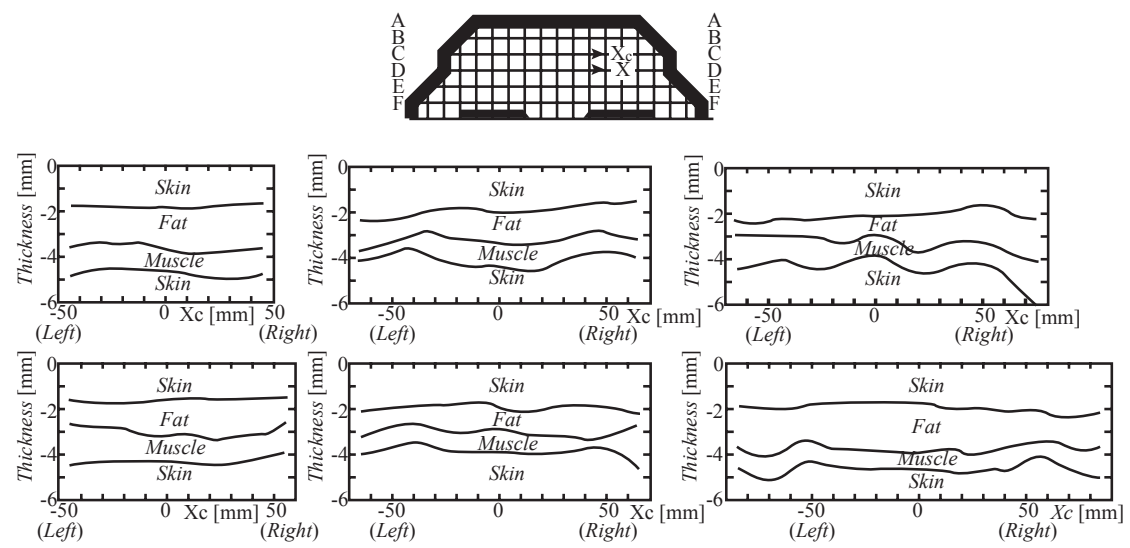

Figure 11: Thickness distribution in forehead.
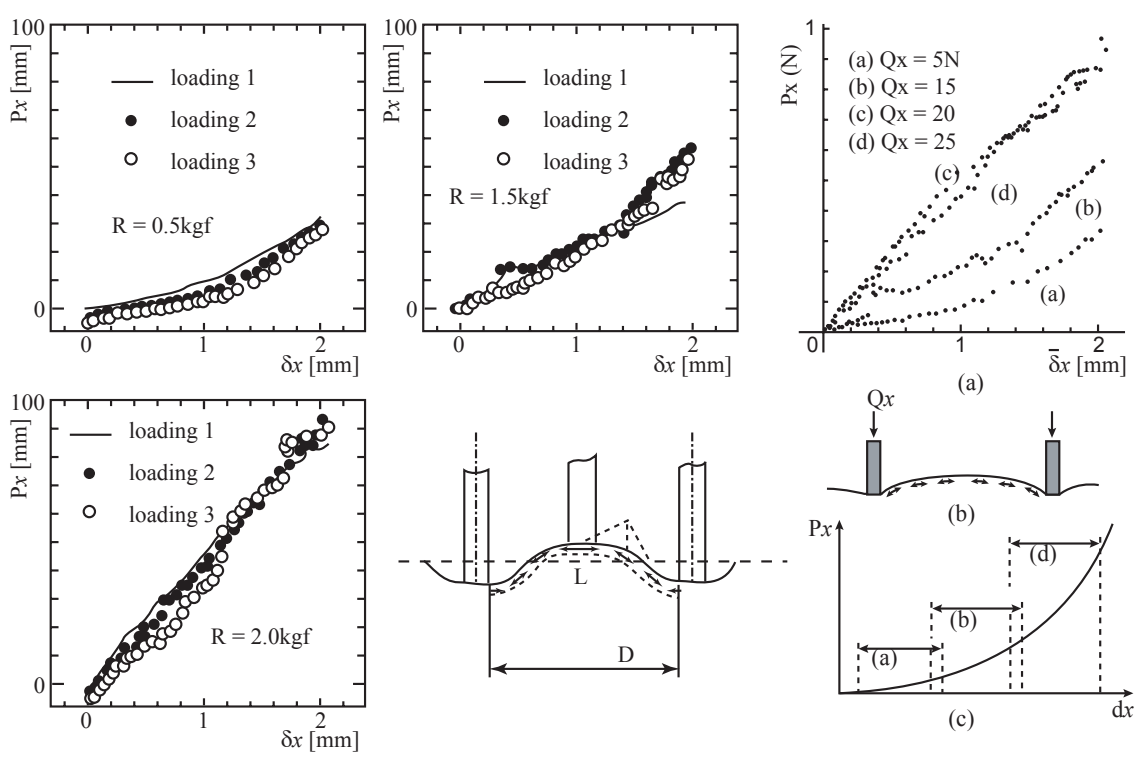

Figure 12: $\quad$ Skin $P_{x}-\delta_{x}$ curve under preloading by outer cylinder.

The loading tests are carried out under the condition that the increment of the displacement is $0.1 \mathrm{~mm}$ and the velocity is $1.25 \mathrm{~mm} / \mathrm{sec}$, which means that the motor rotation increment is equivalent to $1 / 25$ revolution, and the motor speed 30rpm. Figure 12 shows the measured $P_{x}-\delta_{x}$ curves to the running direction of forehead muscle, where the pressing force is varied with four cases from $5 \mathrm{~N}$ to $25 \mathrm{~N}$. The results show that there are four types of $P_{x}-\delta_{x}$ curves, for different pressing conditions of the cylinder, and the significant distinguishments may occur among them as in the figure. 
It is examined and verified that the differences in $P_{x}-\delta_{x}$ curves are mainly induced by the surface traction due to the pressing force as shown in Fig.12(b), and if one slides these $P_{x}-\delta_{x}$ curves according to the amount of the pressing force, the four types of curves are coincident to the unified single $P_{x}-\delta_{x}$ curve as shown in Fig.9(c).

\subsection{Identification of constitutive parameters by analogy with the FEM model}

The FEM model, using 2D isoparametric 20 elements with 69 modes, is constructed as shown in Fig.10(a), fixed at a circular boundary with a diameter of $18 \mathrm{~mm}$.

The calculation results for convergency and similarity of the model A are compared with those obtained by experiment as in Fig.13, and the convergent $P$ $\delta$ curves, coincides well with those obtained by experiment.

Figure 14 shows thus obtained $P_{x}-\delta_{x}, P_{y}-\delta_{y}, T-\theta$ and $P_{y}-\delta_{y}$ curves, obtained by experiments.

In order to compare the aging effect on the $P-\delta$ curve and the difference in constitutive parameters, $P-\delta$ curves for a middle aged man (model B, 54 aged) are also measured in the same way, and the results are shown in the same figure, compared to those of model A. It is seen that higher $P-\delta$ curves are obtained in model B than in model A, and it suggests the hardening of skin due to aging.

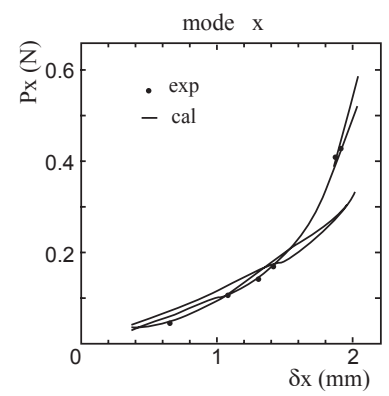

Figure 13: Convergency of identified $P_{x}-\delta_{x}$ curve for model A.

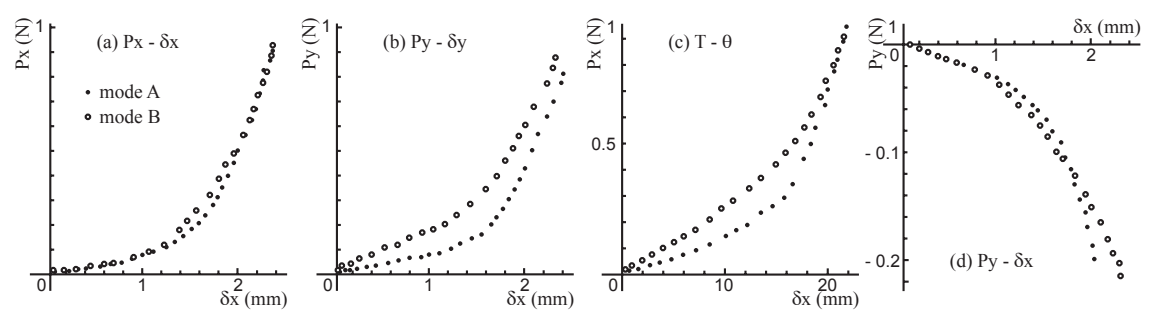

Figure 14: $\quad P_{i}-\delta_{i}$ curve for respective directions. 


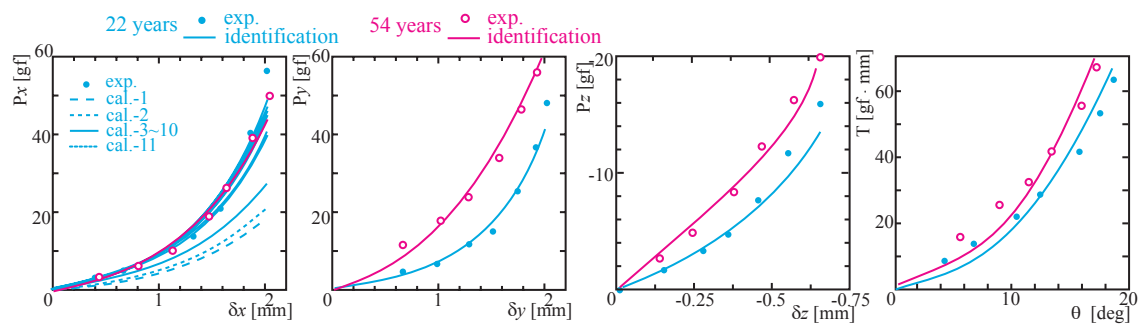

Figure 15.
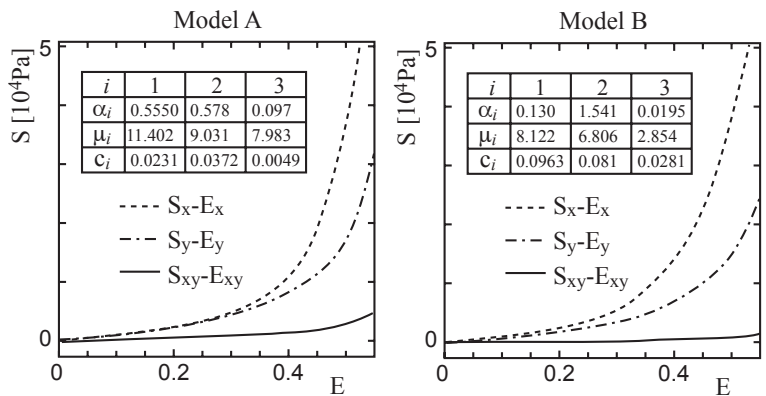

Figure 16: Identified constitutive parameters and stress-strain curves.

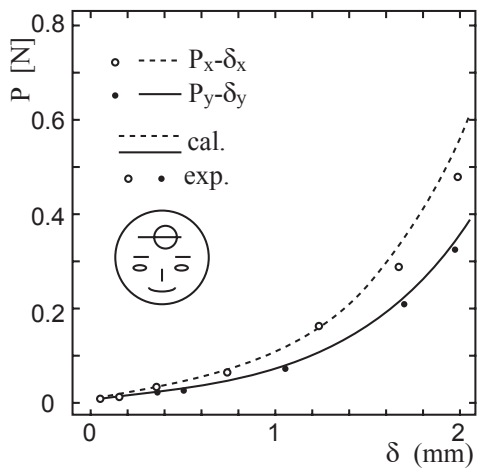

Figure 17: $\quad P-\delta$ curve in case of without load eccentricity.

Figure 16 shows the identified material parameters and Kirchoff stress-Green strain curves for the model A and for the model B, in respective directions.

In order to verify the applicability of the identified parameters shown in the table of Fig.16, other tests, in which the load is applied to the center of the circular region were carried out and the results are compared with those obtained by calculation using the identified material parameters.

It is seen in Fig. 17 that experimental and calculated results successfully coincide well in each other. 


\subsection{Influence of constitutive model due to micro-morphological change by aging}

The aging effect of the constitutive parameter has been examined using by axial tensile test apparatus, with respect to aged 4-month, 10-month and 14 month rabbit skins as shown in Fig. 18 through 21 respectively.

It is seen that along with the aging, the number of cells reduces as in Fig.18, collagen increases as in Fig.20 and elastine decreases as in Fig.19 respectively, so that we can find in Fig.21, the hardness of constitutive model in creases along with the aging.
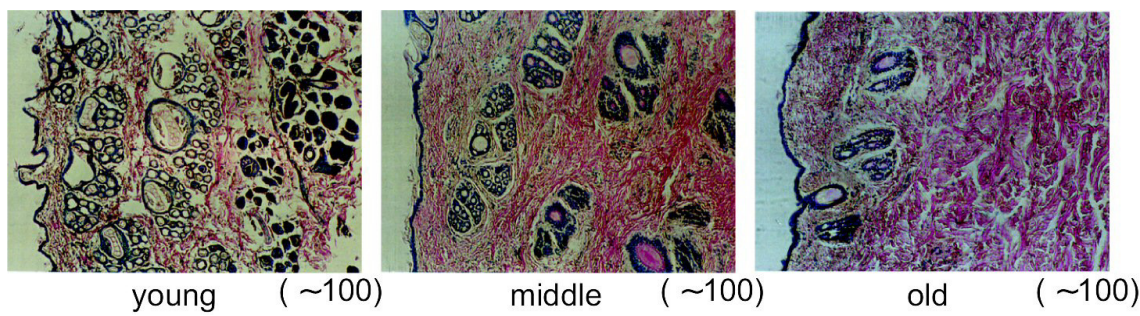

Figure 18: Morphological change in aging.

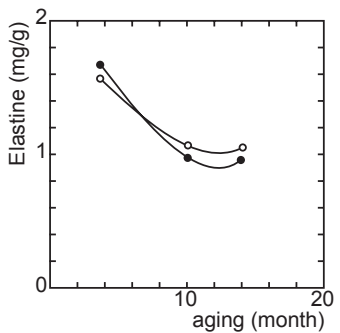

Figure 19: Changes in properties and morphology increases total amount of cycle wrinkling.

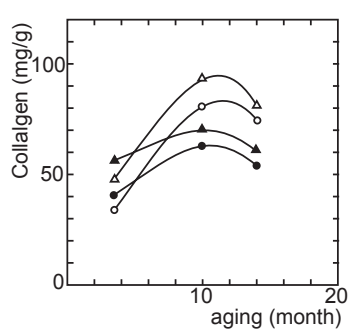

Figure 20: Result of change in chemical composition.

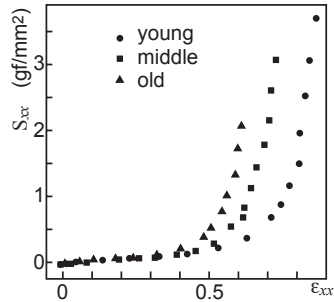

Figure 21: Age effect of $S_{x x}-\varepsilon_{x x}$ curve it require more force than young under same contraction.

\subsection{Identification of material parameters for muscles}

Some eyebrow elevation tests have been carried out by hanging the dropping weight as shown in Fig.22, for detecting the active and passive constitutive characteristics of forehead muscles.

The passive nature of the forehead muscle was obtained at first, by measuring the relation between the increase of weight W and elongation $\left(\Delta L=L_{o^{-}}\right.$ $L$ ), based on the FEM modeling. 
Then, the active nature of the forehead muscle were measured by the relation between E.M.G. measurement of forehead muscle and the eyebrow elevation to the ( $-x$ direction) respectively. The result are summarized as shown in Table 1, based on the least squares approach.

Table 1: $\quad$ Identified material parameters for forehead muscle.

\begin{tabular}{|c|c|c|c|c|c|c|}
\hline Active & $c_{1}$ & $c_{2}$ & $c_{3}$ & $\mu_{1}$ & $\mu_{2}$ & $S_{\max }^{0}$ \\
\hline & 1.04 & 5.82 & 0.894 & 0.689 & 1.45 & 48.6 \\
\hline Passive & $\psi_{1}$ & $\psi_{2}$ & $\psi_{3}$ & $\psi_{4}$ & $C_{m}$ & \\
\hline & 87.0 & 3.30 & 1.88 & 5.78 & 0.808 & \\
\hline
\end{tabular}
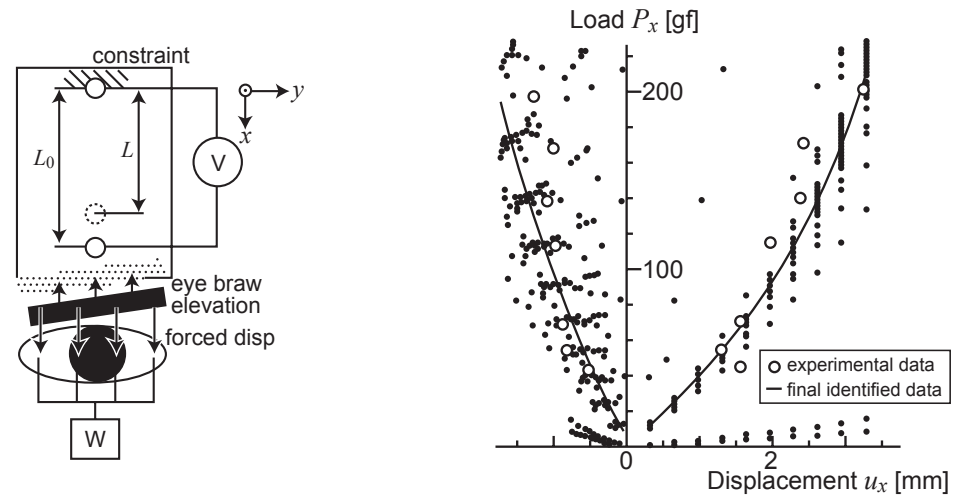

Figure 22: Tests on forehead Figure 23: Identified material parameters of muscles for active and passive material characteristics.
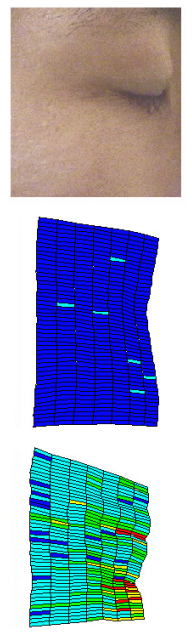

(a)
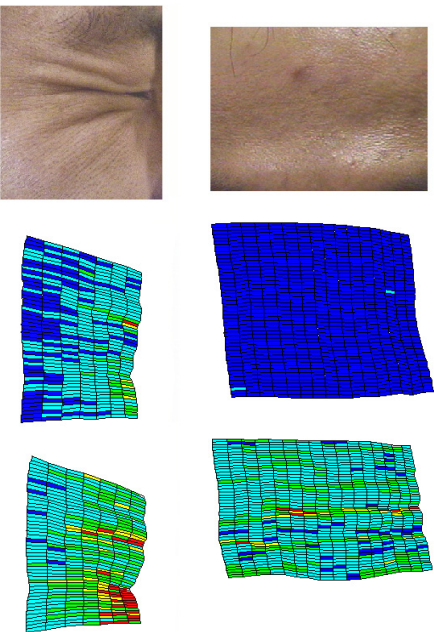

(b)
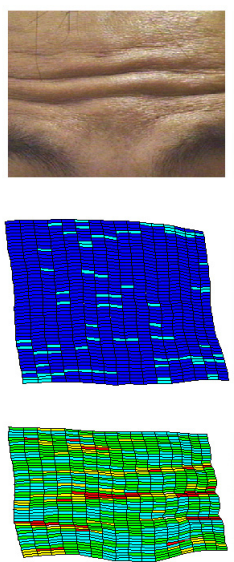

Figure 24 . 
Modelling in Medicine and Biology VII 273
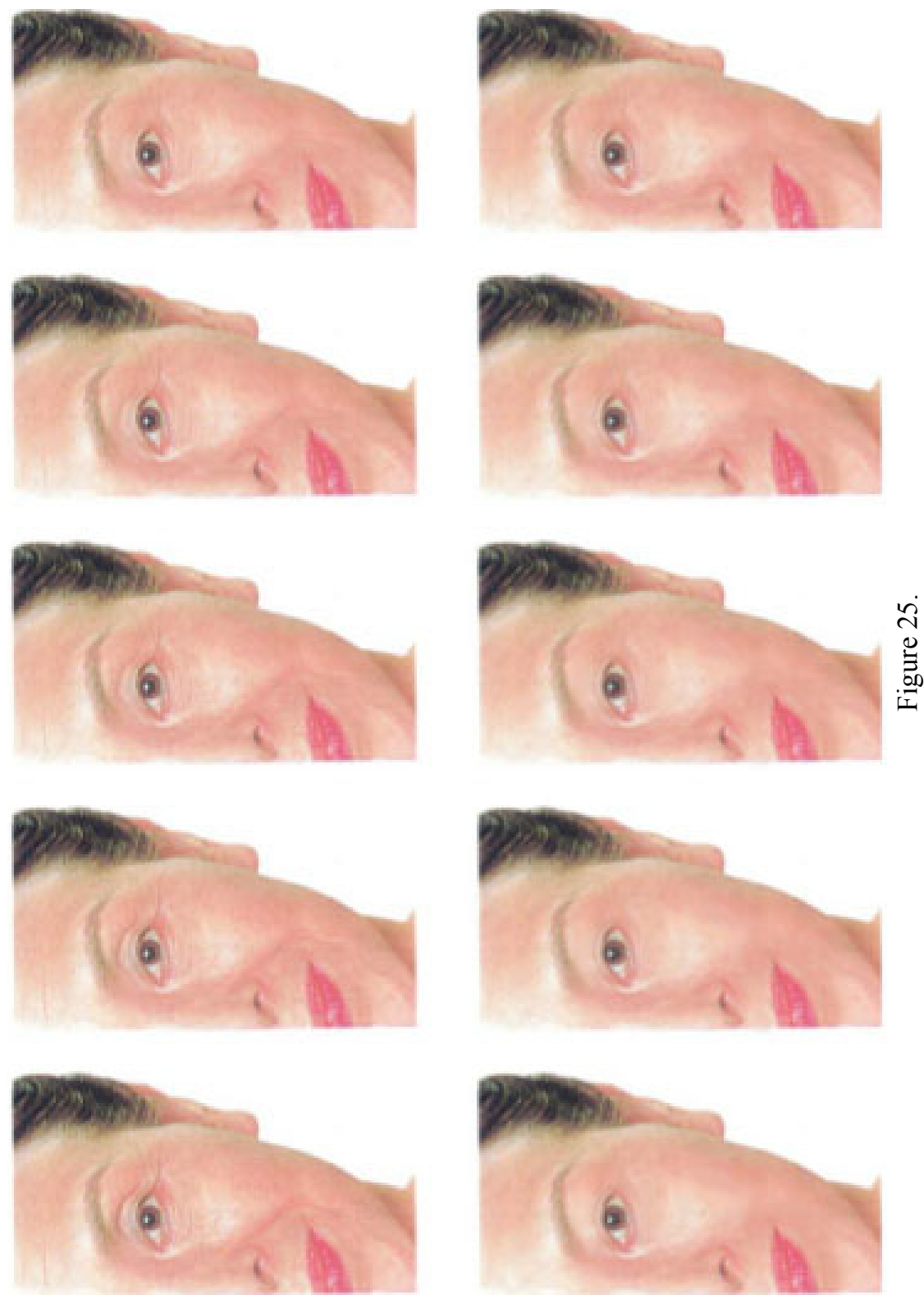

WIT Transactions on Biomedicine and Health, Vol 12, (c) 2007 WIT Press www.witpress.com, ISSN 1743-3525 (on-line) 


\subsection{Application to the facial wrinkling due to aging}

An orbicular muscle contraction during eye-closing gives rise to the eye-crow-'s feet and the associated wrinkling stress as shown in Fig.24 (a).

The contraction of forehead muscle give rise to the horizontal wrinkling in forehead skin and the associated wrinkling stress as shown in Fig.24 (b).

The iterative wrinklings during long time history so far stated give rise to the permanent wrinkling as shown in Fig.25.

We can conclude that such permanent wrinkling problems can be analysed, using so far stated mechanics of modeling.

\section{Concluding remarks}

An automatic loading and sensing system for obtaining in vivo multi-axial $P_{i}-\delta_{i}$ curves in facial skin of living human has been developed.

The constitutive parameters of the Kirchoff stress-Green strain relation have been successfully determined by combining it with FEM computer modeling, using the least squares approach.

The application to some forehead skins of actual humans aged 22 and 54 years has proven the satisfactory applicability of the method developed.

\section{References}

[1] Y. C. Fung, Biomechanics: mechanical properties of living tissues (Spriger-Verlag, 1993).

[2] Y. Lanir and Y. C. Fung, Two-dimensional mechanical properties of rabbit skin-I. Experimental system, J. Biomechanics, 7, 1974, 29-34.

[3] Y. Lanir and Y. C. Fung, Two-dimensional mechanical properties of rabbit skin-II. Experimental results, J. Biomechanics, 7, 1974, 171-182.

[4] T. Tsuta, M. Li, T. Iwamoto, T. Kumagai and O. Kaneko, Muscle's continuum mechanics and FEM simulation on facial expression for muscle-soft tissue combined system, Faculty of Engineering, Hiroshima University, (in Japan), 46-1,1997.

[5] M. Sato, T. Ohashi, H. Abe and T. Matsumoto, Measurement of nonlinear elastic properties in aortic walls using pipette aspiration technique under biaxial stretching, Proc. International Conf. on New Frontiers in Biomechanical Engineering, Tokyo, Japan, 1997, 81-82.

[6] T. Tsuta, C. Wang and K. Yamane, Computer simulation of facial expression (1st report, finite deformation analysis of hyperelastic softtissue by the finite element method, and identification of mechanical properties), Trans. JSME, (in Japan), 58-551, 1992, 1107-1114.

[7] D. A. Hoeltzel, P. Altman and K. Buzard, Strip extensionmetry for comparison of the mechanical response of bovine rabbit and human corneas, J. Biomech. Eng., 114, 1992, 202-21 